\title{
Reduced cortical volume and thickness and their relationship to medical and operative features in post-Fontan children and adolescents
}

\author{
Christopher G. Watson ${ }^{1,2}$, Christian Stopp ${ }^{3}$, David Wypij ${ }^{3,4}$, Jane W. Newburger ${ }^{3,5}$ and Michael J. Rivkin ${ }^{2,6,7,8}$
}

BACKGROUND: We compared brain cortical and subcortical gray matter volumes and cortical thickness between postFontan patients and healthy controls, and examined brain anatomical associations with operative and medical history characteristics.

METHODS: Post-Fontan ( $n=128$ volumes; $n=115$ thickness) and control subjects ( $n=48$ volumes; $n=45$ thickness) underwent brain MRI at ages 10-19 y. Subcortical and cortical volumes and cortical thicknesses were measured for intergroup comparison. Associations between brain measures and clinical measures were assessed in the Fontan group.

RESULTS: Widespread, significant reduction in brain volumes and thicknesses existed in the Fontan group compared to controls, spanning all brain lobes and subcortical gray matter. Fontan subjects treated with vs. without the Norwood procedure had smaller volumes in several terminal clusters, but did not differ in cortical thickness. Older age at first operation and increasing numbers of cardiac catheterizations, operative complications, and catheterization complications were associated with lower regional volumes and thicknesses. Increasing numbers of operative complications and cardiac catheterizations were associated with smaller regional volumes in the Norwood group.

CONCLUSION: The post-Fontan adolescent brain differs from the normal control brain. Some of these differences are associated with potentially modifiable clinical variables, suggesting that interventions might improve long-term neurocognitive outcome.

C ongenital heart disease (CHD) is the most common congenital anomaly, with single ventricle (SV) defects occurring in nearly $10 \%$ of patients with CHD (1). Patients with SV, particularly hypoplastic left heart syndrome (HLHS), have higher mortality and morbidity than those with other types of CHD, but their survival has steadily increased for several decades (2). The Fontan procedure, as well as staged palliation via the Norwood procedure for HLHS, rep resented major steps forward in the care of patients with SV defects (3). Prior to the establishment of these procedures, survival was low; in HLHS patients, survival to $1 \mathrm{y}$ of age was virtually $0 \%$ (4). More recently, the Single Ventricle Reconstruction Trial found that overall 3-y transplant-free survival in HLHS patients was 64\%. Among those who survive to Fontan in the current era, however, 10 -y survival is over $90 \%(5,6)$. Consequently, focus has turned progressively toward neurologic injury and its effects on neurodevelopmental outcomes in the aging SV population. Studies of the burgeoning SV population now reaching adulthood have revealed a more complete picture of their longterm neurodevelopment, including cognitive deficits similar to those seen in other CHD, but greater in severity $(7,8)$. The structural correlates of these cognitive and neurodevelopmental deficits have not been fully defined.

Differences in brain structure of CHD patients have been documented in utero (9). Even prior to initial surgery, which often occurs in the neonatal period, signs of brain injury and abnormal cerebral hemodynamics are apparent both in autopsy and MRI studies $(10,11)$. Indeed, significant reduction in brain cortical volume has been observed recently in a heterogeneous group of neonates with CHD (12). Early postoperative MRI often shows new or more severe brain lesions (13). Patients with SV require multiple surgeries, often beginning with the stage 1 Norwood procedure in the first few weeks of life, and leading to the Fontan procedure generally between 1.5 and $4 \mathrm{y}$ of age (3). Post-Fontan brain MRI studies of SV patients are few, but suggest that evidence of brain injury is still present in some patients into childhood $(14,15)$. Recently, we reported increased number of gross anatomic brain abnormalities by MRI in our current sample of SV patients 10-19 $y$ of age as compared to a referent sample (7). Accompanying these differences in brain structure are deficits in several cognitive and behavioral domains including attention, memory, executive function, motor function, and visual-spatial processing. However, quantitative analyses of specific brain structural

\footnotetext{
'Graduate Program for Neuroscience, Boston University, Boston, Massachusetts; ${ }^{2}$ Department of Neurology, Boston Children's Hospital, Boston, Massachusetts; ${ }^{3}$ Department of Cardiology, Boston Children's Hospital, Boston, Massachusetts; ${ }^{4}$ Department of Biostatistics, Harvard T.H. Chan School of Public Health, Harvard Medical School, Boston, Massachusetts; ${ }^{5}$ Department of Pediatrics, Harvard Medical School, Boston, Massachusetts; ${ }^{6}$ Department of Radiology, Boston Children's Hospital, Boston, Massachusetts; ${ }^{7}$ Department of Psychiatry, Boston Children's Hospital, Boston, Massachusetts; ${ }^{8}$ Department of Neurology, Harvard Medical School, Boston, Massachusetts. Correspondence: Michael J. Rivkin (michael.rivkin@childrens.harvard.edu)

Received 21 August 2016; accepted 15 January 2017; advance online publication 3 May 2017. doi:10.1038/pr.2017.30
} 


\section{Articles Watson etal.}

differences in children and adolescents with SV have not been conducted.

We acquired volumetric brain MRI data to compare cortical and subcortical gray matter volumes and cortical thicknesses between post-Fontan and healthy control children and adolescents. We also sought to examine associations of perioperative and medical history characteristics with these brain structure measures. Additionally, we considered group comparisons based on a stratification of the Fontan subjects into those children who underwent the Norwood procedure as their first operation and those who did not.

\section{METHODS \\ Subjects}

Study subjects were enrolled at Boston Children's Hospital between 2010 and 2012. Inclusion criteria for the Fontan group were (i) age $10-19 \mathrm{y}$ at the time of enrollment, (ii) diagnosis of single ventricle, and (iii) cardiac surgery including Fontan procedure, Fontan re-do, or other open heart surgery at least 6 mo before testing. Exclusion criteria included presence of objects that are contraindications of MRI (e.g., pacemaker, metal implants). Clinical data were obtained through review of medical records, questionnaires, and family interview.

Inclusion criteria for the control group of healthy adolescents were adapted from the NIH MRI study of normal brain development (16). Children with known risk factors for brain disorders (e.g., intra-uterine exposure to toxicants, histories of closed head injury with loss of consciousness, language disorder or Axis 1 psychiatric disorder, first degree relative with a lifetime history of an Axis 1 psychiatric disorder, inherited neurologic disorder, or abnormality on neurological examination) were excluded. We also excluded control subjects for whom MRI was contraindicated, those with Trisomy 21, sickle cell anemia, muscular dystrophy, adolescents with other forms of CHD requiring surgical correction, and subjects whose primary language was not English. Head circumference measurements at birth were not available for all subjects and so were not included in data collection. This study was approved by the Institutional Review Board and adhered to institutional guidelines. Parents and subjects over 18 $\mathrm{y}$ of age provided informed consent, and subjects less than $18 \mathrm{y}$ of age provided assent.

\section{MRI Acquisition}

Subjects were scanned on one of two systems at Beth Israel Deaconess Medical Center: a 3 Tesla (T) General Electric (GE) Signa system or, for patients who possessed implanted intrathoracic cardiovascular devices or coils, a 1.5T GE Twinspeed scanner (General Electric Medical Systems, Milwaukee WI). We acquired a T1-weighted 3D magnetization-prepared rapid gradient echo (MP-RAGE) sequence with parameters: TR $/ \mathrm{TE}=7 \mathrm{~ms} / 2.8 \mathrm{~ms}$; flip angle $=8^{\circ}$; voxel size $=$ $1 \times 1 \times 1 \mathrm{~mm}^{3}$. An 8 -channel phased-array head coil was used on both the $1.5 \mathrm{~T}$ and $3 \mathrm{~T}$ scanners.

\section{MRI Analysis and Anatomic Classification}

Images were processed using Freesurfer v5.3 (A.A. Martinos Center for Biomedical Imaging, Massachusetts General Hospital). The technical details are described elsewhere (17). Briefly, this procedure involves partitioning volumetric MRI images into white matter, gray matter, and cerebrospinal fluid. The deep gray matter in each hemisphere is segmented into seven discrete subcortical structures, and their volumes are calculated: caudate, globus pallidus, putamen, thalamus, hippocampus, amygdala, and nucleus accumbens. Next, the outer pial surface of the brain is delineated, as is the surface comprising the white matter/gray matter junction. Cortical thickness is obtained by taking the distance (in $\mathrm{mm}$ ) between these two surfaces. Finally, the cortical surface is parcellated into discrete units based on gyral and sulcal anatomy. We used the Desikan-Killiany-Tourville (DKT) atlas to obtain mean cortical volume and thickness for 31 gyri in each hemisphere (18). The ventricles, cerebellum, and brainstem were not included in analyses. All images were inspected for image processing errors and manually corrected when necessary.

Brain regions of interest were grouped based on distance from the main cerebral arterial trunk supplying their blood based on standard nomenclature (i.e., proximal, middle, or terminal) (19). Combining left and right hemispheric regions to reduce multiple comparisons resulted in 24 subcortical and cortical volume clusters and 20 cortical thickness clusters (Table 1) (20). We additionally performed a surface-based vertex-wise analysis of between-group cortical thickness differences in order to preserve spatial specificity. First, data from each subject was resampled to a standard space with the MNI305 template; this has been validated in children previously (21). Next, each dataset was spatially smoothed using a $15 \mathrm{~mm}$ full-width at half maximum (FWHM) Gaussian kernel along the cortical surface (22). Then, a general linear model (GLM) was fit at each vertex (i.e., data point), adjusting for age at MRI and sex. MRI field strength was excluded due to the lack of any significant main or interaction effects in a GLM when including it as a covariate. Each hemisphere was analyzed separately, and statistical significance was determined using the false discovery rate (FDR) with $q<0.025$ to adjust for multiple comparisons across vertices and both hemispheres (23).

\section{Statistics}

Preoperative and sociodemographic variables comprised sex, race, ethnicity, birth weight, gestational age, concurrent family social

Table 1. Volume and thickness clusters ${ }^{\mathrm{a}}$

Distance from arterial trunk

Proximal

Pericalcarine

Caudal anterior cingulate + rostral anterior cingulate

Superior temporal + transverse temporal

Middle

Caudate

(volumes only)

Caudal middle frontal

Precentral + postcentral

Superior parietal + paracentral

Superior frontal + rostral middle frontal

Inferior parietal

Middle temporal

Pars opercularis

Terminal

Pallidum + putamen

(volumes only)

Medial orbitofrontal

Isthmus cingulate + posterior cingulate

Pars orbitalis + pars triangularis

Thalamus + hippocampus

(volumes only)

Entorhinal

Precuneus + cuneus + lingual

Parahippocampal

Amygdala

(volumes only)

Nucleus accumbens + lateral orbitofrontal ${ }^{b}$

Fusiform + inferior temporal + lateral occipital

Insula

Supramarginal

${ }^{a}$ All regions are bilateral. ${ }^{b}$ For thickness cluster, lateral orbitofrontal only. 
status, and age at MRI. Operative and medical history measures for Fontan subjects included Norwood status; genetic abnormality; age at first operation; open first operation (vs. shunt); deep hypothermic circulatory arrest duration and total support duration at first operation; complications at first operation; total operations, open operations, operative complications, catheterizations, and catheterization complications; and incidence of seizure (clinical or EEG) or any neurological event (i.e., stroke, seizure, choreoathetosis, and meningitis). Field strength of MRI and total intracranial volume (TIV) served as covariates for adjustment purposes and descriptive statistics (i.e., means and standard deviations) of two measures of intelligence are also described. Group comparisons of sociodemographic, operative and medical history characteristics, intelligence measures, and structural MRI findings, were performed using Fisher exact tests for categorical measures and analysis of variance or Wilcoxon tests for continuous measures with normal or non-normal distributions, respectively. Group comparisons of intelligence measures also adjusted for the type of age-based assessment, i.e., the Wechsler Intelligence Scale for Children for subjects age $<17 \mathrm{y}$ and the Wechsler Adult Intelligence Scale for subjects age $\geq 17 \mathrm{y}$.

Linear regression was used to compare: (i) cortical volumes $\left(\mathrm{mm}^{3}\right)$ of Fontan and control adolescents with adjustment for age at MRI, sex, MRI field strength, and TIV; and (ii) cortical thickness (mm) of Fontan and control adolescents with adjustment for age at MRI, sex, and field strength. TIV was excluded as a covariate in analyses of cortical thickness because it has been shown that this adjustment is inappropriate (24). We also assessed differences between patients stratified by Norwood status (hereafter referred to as "Norwood" and "non-Norwood"), and between these subgroups and controls, with similar adjustments, as well as assessing differences between patients stratified by presence of focal infarction or atrophy. Partial Spearman correlations, with adjustment for the same factors as outlined for the regressions, were used to examine the associations of mean regional cortical volumes and thicknesses of the Fontan patients with their operative and medical history characteristics. The significance criterion for group differences was $P<0.05$ and for correlations was $P<$ 0.01 .

\section{RESULTS}

\section{Subjects}

Table 2 presents the medical and demographic characteristics of the Fontan and control groups. Anatomic MRIs were completed for 144 Fontan subjects. The majority of Fontan subjects had a lateral tunnel fenestrated Fontan ( $n=129,90 \%)$; others were extracardiac fenestrated $(n=8,6 \%)$, extracardiac nonfenestrated $(n=5,3 \%)$, and lateral tunnel nonfenestrated $(n=1,1 \%)$. Among those with MRIs, eight failed postimaging quality control due to movement artifacts, two from poor tissue contrast, five from foreign body artifacts, and one from a large stroke affecting image processing, yielding 128 Fontan subjects $(76 \mathrm{M}, 52 \mathrm{~F}$; mean age $=14.6 \pm 2.9 \mathrm{y}$; range $=10.2-$ $19.96 \mathrm{y}$ ) for the final volume analysis. Patients in the Norwood subgroup $(n=50 ; 34 \mathrm{M}, 16 \mathrm{~F}$; mean age $=14.4 \pm 2.8 \mathrm{y})$ did not differ from non-Norwood patients $(n=78 ; 42 \mathrm{M}, 36 \mathrm{~F}$; mean age $=14.8 \pm 3.0 y)$ in age at MRI, sex, field strength of scanner, or total intracranial volume (TIV). For the cortical thickness analysis, an additional 13 Fontan subjects were excluded due to movement artifacts, yielding 115 Fontan subjects (68 M, 47 F; mean age $=14.7 \pm 3.0 \mathrm{y}$; range $=10.2-19.96 \mathrm{y}$ ). Fontan subjects used in the volume analysis had fewer total operative complications than those who were not used (median: 2, range: 0-11 vs. median: 4 , range: $0-12 ; P=0.03$ ); Otherwise, Fontan subjects included in the final volume and thickness analyses were comparable in demographic characteristics to those excluded.
Among 50 healthy controls scanned, data from 2 were excluded due to movement artifact, yielding 48 healthy controls $(28 \mathrm{M}, 20 \mathrm{~F}$; age $=15.4 \pm 2.5 \mathrm{y}$, range $=10.1-19.7 \mathrm{y})$ in the volume analysis. The cortical thickness analysis included 45 control subjects $(26 \mathrm{M}, 19 \mathrm{~F}$; mean age $=15.5 \pm 2.5 \mathrm{y}$; range $=10.1-19.7 \mathrm{y}$ ) as data from 5 were excluded due to movement artifact. Controls used in the thickness analysis had higher social status (median: 53.5 , range: $32-66$ vs. median: 44 , range: $40-50 ; P=0.02$ ) than those who were excluded. A significantly lower proportion of Fontan adolescents were scanned at 3T compared to controls $(P=0.004)$. Finally, clear differences in cognitive measures emerged among the clinical groups imaged. Fontan subjects had significantly reduced Full Scale IQ (FSIQ) scores as compared to controls (Fontans 92.2 \pm 16.3 ; Controls $109.2+11.8 ; P<0.001$; Table 2). Additionally, Norwood patients demonstrated significantly lower performance than non-Norwood patients (non-Norwoods $95.3+15.3$; Norwoods $87.3+16.8 ; P=0.006$; Table 2). Similar reductions were found in Verbal Comprehension IQ between Fontan and Controls (Fontans 98.1 + 14.9; Controls $109.2+12.3$; $P<0.001$; Table 2) as well as for Norwood patients as compared to non-Norwood patients (non-Norwoods $101.7+14.2$; Norwoods $92.5+$ 14.5; $P<0.001$; Table 2). Comprehensive neuropsychological characteristics of these clinical groups have been reported previously (3). Correlational analyses between brain volumetric measures and global cognitive measures are currently in progress and will be reported separately.

\section{Gross Structural Imaging Characteristics}

Table 3 lists the gross structural brain MRI findings of the Fontan and control groups. A significantly greater proportion of Fontan subjects than control subjects demonstrated abnormalities (65 vs. $11 \% ; P<0.001$ ), predominantly due to the difference in the incidence of hemosiderin deposit (55 vs. 3\%; $P<$ $0.001)$. Focal infarction or atrophy was also significantly more frequent among Fontan subjects than controls (12 vs. 0\%; $P$ $=0.01$ ). The groups did not differ in presence of diffuse, generalized, or developmental abnormalities. No differences were observed in structural MRI findings between groups when stratifying by occurrence of Norwood procedure.

\section{Volumetric Findings}

Subcortical and cortical volume differences. TIV was significantly lower in Fontan adolescents compared to controls $\left(1,431\right.$ vs. $\left.1,524 \mathrm{~cm}^{3} ; P<0.001\right)$. Among the 24 volume clusters tested, 7 (29\%) clusters showed significantly smaller volumes among Fontan subjects relative to those of healthy controls (Figure 1a,b and Table 4): (i) superior temporal-transverse temporal; (ii) inferior parietal; (iii) pallidum-putamen; (iv) thalamus-hippocampus; (v) precuneus-cuneus-lingual; (vi) amygdala; and (vii) fusiform-inferior temporal-lateral occipital regions. Relative to controls, Norwood subjects demonstrated as many regions with significant volume reduction as compared to non-Norwood subjects (6 vs. 3; Table 4). Further, Norwood subjects had smaller volume than non-Norwood subjects in several terminal clusters: (i) 


\section{Articles | watson e tal.}

Table 2. Demographic and medical characteristics of Fontan and control subjects with volumetric MRI data

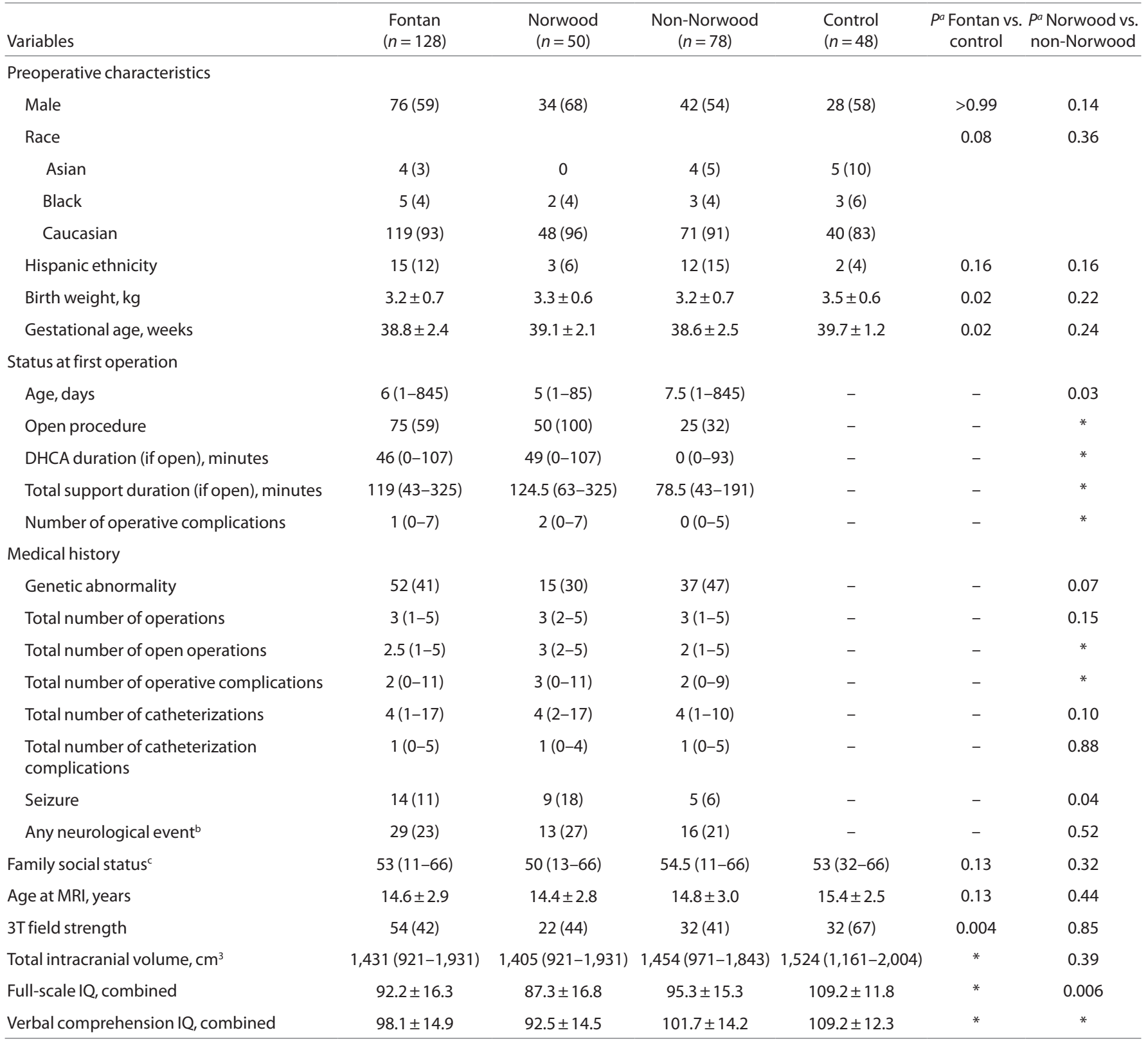

\footnotetext{
Values are $n(\%)$, mean \pm SD, or median (range).
}

${ }^{a}$ Determined by Fisher exact test for categorical variables, analysis of variance for continuous variables represented with means, and Wilcoxon test for continuous variables represented with medians. Full-scale and verbal comprehension IQ comparisons adjust for type of age-based assessment. bIncludes stroke, seizure, choreoathetosis, and meningitis.

cScore on Hollingshead Four Factor Index of Social Status, with higher scores indicating higher social status. ${ }^{*} P<0.001$.

pallidum-putamen; (ii) isthmus cingulate-posterior cingulate; (iii) thalamus-hippocampus; (iv) entorhinal; and (v) amygdala regions. Finally, volume of caudal middle frontal and medial orbitofrontal regions was found to be significantly smaller among Fontan adolescents with evidence of focal infarction or atrophy as compared to Fontan adolescents with no focal infarction or atrophy after adjusting for age at MRI, sex, field strength, and TIV (caudal middle frontal: 6,849 \pm 360 vs. $7,720 \pm 123, P=0.04$; medial orbitofrontal: $4,751 \pm 127$ vs. $5,091 \pm 63, P=0.04)$.
Cortical thickness differences. Among the 20 thickness clusters tested, $10(50 \%)$ were significantly thinner in the Fontan group than the control group (Figure 1c,d and Table 5): (i) caudal middle frontal; (ii) precentral-postcentral; (iii) superior parietal-paracentral; (iv) superior frontal-rostral middle frontal; (v) inferior parietal; (vi) middle temporal; (vii) pars orbitalis-pars triangularis; (viii) precuneus-cuneuslingual; (ix) parahippocampal; and (x) fusiform-inferior temporal-lateral occipital regions. Norwood subjects did not differ in cortical thickness for any cluster from non-Norwood 
Table 3. Structural MRI findings of Fontan and control subjects with volumetric MRI data ${ }^{a}$

\begin{tabular}{|c|c|c|c|c|c|c|}
\hline Variables & $\begin{array}{l}\text { Fontan } \\
(n=128)\end{array}$ & $\begin{array}{c}\text { Norwood } \\
(n=50)\end{array}$ & $\begin{array}{c}\text { Non-Norwood } \\
\quad(n=78)\end{array}$ & $\begin{array}{l}\text { Control } \\
(n=48)\end{array}$ & $\begin{array}{l}P^{b} \text { Fontan } \\
\text { vs. control }\end{array}$ & $\begin{array}{l}P^{b} \text { Norwood vs. } \\
\text { non-Norwood }\end{array}$ \\
\hline Any abnormality & $64 / 98(65)$ & $27 / 38(71)$ & $37 / 60(62)$ & $4 / 38(11)$ & * & 0.39 \\
\hline Focal or multifocal abnormality & $59 / 98(60)$ & $24 / 38(63)$ & $35 / 60(58)$ & $1 / 38(3)$ & * & 0.68 \\
\hline Focal infarction or atrophy & $15 / 128(12)$ & $4 / 50(8)$ & $11 / 78(14)$ & $0 / 48(0)$ & 0.01 & 0.40 \\
\hline Brain mineralization/iron deposit & $54 / 98(55)$ & $22 / 38(58)$ & $32 / 60(53)$ & $1 / 38(3)$ & * & 0.68 \\
\hline Ventriculogealy & $2 / 128(2)$ & $1 / 50(2)$ & $1 / 78(1)$ & $0 / 48(0)$ & $>0.99$ & $>0.99$ \\
\hline Abnormal $\mathrm{T} 2$ hyperintensities & $9 / 123(7)$ & $5 / 47(11)$ & $4 / 76(5)$ & $2 / 48(4)$ & 0.73 & 0.30 \\
\hline Generalized abnormality & $0 / 128(0)$ & $0 / 50(0)$ & $0 / 78(0)$ & $0 / 48(0)$ & - & - \\
\hline Developmental abnormality & $5 / 128(4)$ & $1 / 50(2)$ & $4 / 78(5)$ & $2 / 48(4)$ & $>0.99$ & 0.65 \\
\hline \multicolumn{7}{|c|}{$\begin{array}{l}\text { Values are } n(\%) \text {. } \\
\text { The denominators in the table entries differ from the total group size because some subjects did not complete the full set of MRI sequences needed to identify some of the brain } \\
\text { abnormality types. } \\
\text { bDetermined by Fisher exact test. } \\
\text { cMinor malformations include arachnoid cyst, absent septal leaflets, pericallosal lipoma, and periventricular cycts in the left and right frontal lobes in the Fontan group; and } \\
\text { polymicrogyria and pineal cyst in referent group. } \\
{ }^{*} P<0.001 \text {. }\end{array}$} \\
\hline
\end{tabular}

subjects. Interestingly, thickness in entorhinal and insular regions was found to be significantly lower among Fontan adolescents with evidence of focal infarction or atrophy after adjusting for age at MRI, sex, and field strength (entorhinal: $3.07 \pm 0.06$ vs. $3.24 \pm 0.03, P=0.03$; insular: $3.03 \pm 0.03$ vs. $3.13 \pm 0.01, P=0.03)$.

Vertex-wise cortical thickness differences. Figure 1e shows the areas in which cortical thickness was significantly thinner in the Fontan group compared to the control group. Differences were distributed similarly across both hemispheres, predominantly in posterior medial and lateral cortices, but also in lateral frontal cortices. Specifically, portions of the caudal middle frontal gyrus, precentral gyrus, superior parietal lobe, paracentral lobule, rostral middle frontal gyrus, inferior parietal lobe, precuneus, lingual gyrus, and fusiform gyrus showed bilateral involvement. In addition, left middle temporal gyrus and right lateral occipital gyrus contained clusters of cortical thickness differences. These regions largely overlap with the group differences in the regions of interest listed in Table 5.

\section{Volumetric Correlations With Operative Characteristics and Medical History}

Correlations with volume. No significant associations were noted between cortical or subcortical volumes and characteristics of the reported first operation among all Fontan subjects, nor among non-Norwood subjects. However, among Norwood subjects, longer total support duration was associated with lower caudal middle frontal volume $(r=-0.48, P=0.003)$.

For associations with medical history variables, increasing numbers of open operations and operative complications were associated with lower thalamus-hippocampus volume
( $r=-0.26, P=0.004$ and $r=-0.24, P=0.008$, respectively) among all Fontan subjects. Increasing number of catheterization complications was associated with lower volume of the medial orbitofrontal and parahippocampal clusters $(r=-0.26$, $P=0.007$ and $r=-0.26, P=0.006$, respectively).

Among Norwood subjects, increasing number of open operations was associated with higher entorhinal volume $(r=0.46$, $P=0.003)$. Increasing number of operative complications was associated with lower isthmus cingulate-posterior cingulate ( $r=-0.42, P=0.007)$, fusiform-inferior temporal-lateral occipital $(r=-0.48, P=0.002)$, and insula $(r=-0.46, P=$ 0.003 ) volumes. Increasing number of catheterizations was associated with lower medial orbitofrontal volumes $(r=-0.41$, $P=0.008)$. Among non-Norwood subjects, increasing number of open operations was associated with lower inferior parietal volume $(r=-0.36, P=0.003)$ and increasing number of catheterization complications was associated with lower parahippocampal volume $(r=-0.41, P<0.001)$.

Correlations with thickness. Among all Fontan subjects, older age at first operation was associated with lower caudal middle frontal $(r=-0.25, P=0.008)$, middle temporal $(r=-0.28, P$ $=0.003)$, and pars orbitalis-pars triangularis $(r=-0.25, P=$ 0.007 ) thickness. Additionally, increasing number of catheterizations was associated with lower isthmus cingulate-posterior cingulate cortical thickness $(r=-0.26, P=0.005)$ as well as lower fusiform-inferior temporal-lateral occipital cluster thickness $(r=-0.25, P=0.009)$. Open operation was associated with higher caudal anterior cingulate - rostral anterior cingulate thickness in all Fontan subjects $(r=0.28, P=0.003)$ as well as in non-Norwood subjects ( $r=0.33, P=0.006)$. Also among non-Norwood subjects, increasing total support duration was 


\section{Articles}
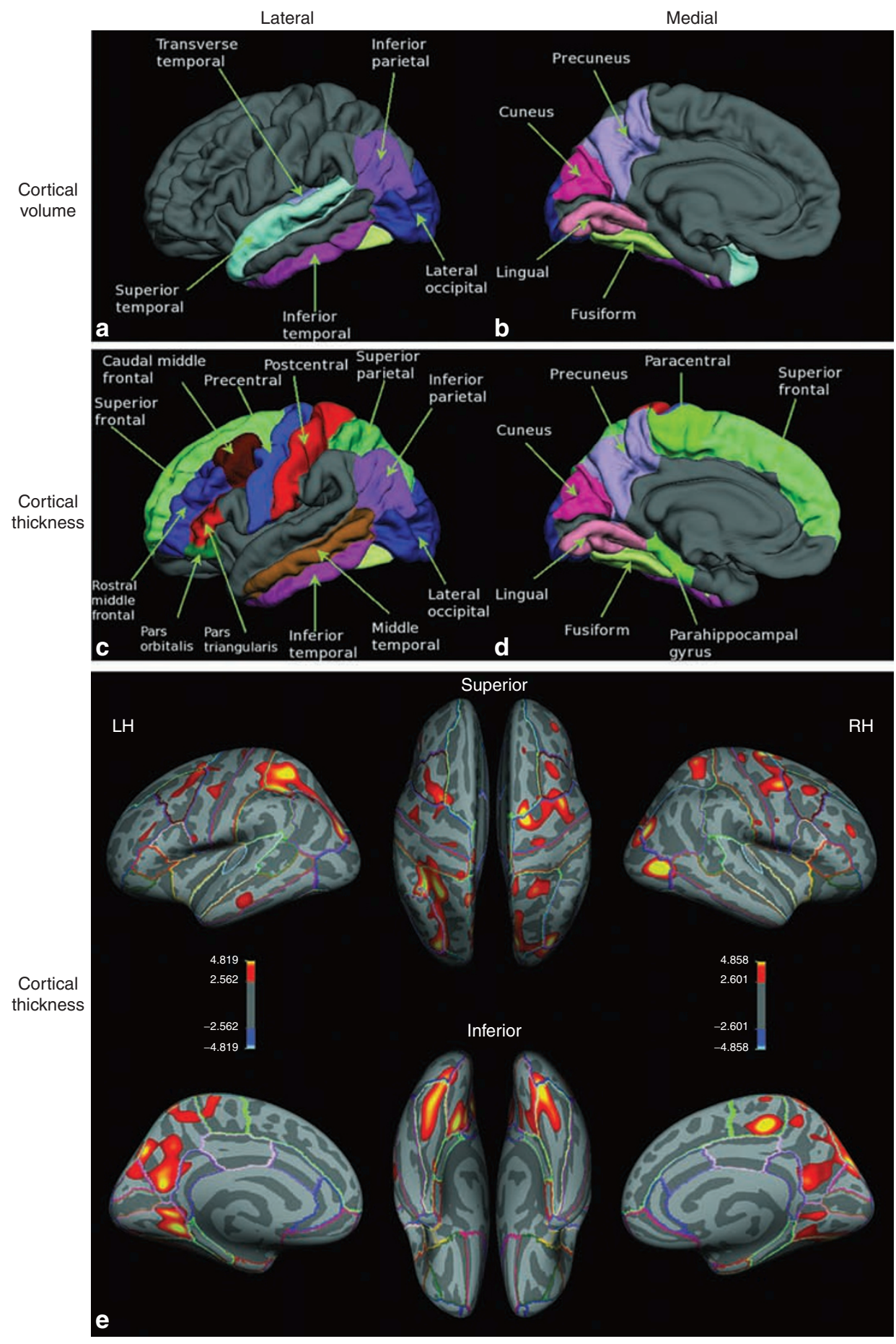

Figure 1. Regions of cortical volume and thickness differences between Fontan and control groups. (a) Lateral and (b) medial views of the cortical surface showing locations in non-gray color of reduced mean regional volume in the Fontan group compared to the control group. (c) Lateral and (d) medial views of the cortical surface showing locations in non-gray color of reduced mean cortical thickness in the Fontan group compared to the control group. Regional volumes and thicknesses in a-d reflect combination of left and right hemispheric regions to reduce multiple comparisons. (e) Surfacebased vertex-wise analysis of cortical thickness differences between the Fontan group and the control group. Regions in which cortical thickness was significantly lower in the Fontan group as compared to the control group are depicted in red-yellow. The data are displayed on an "inflated" surface which allows for visualization of sulcal regions which are not visible on standard inspection due to cortical folding. Brain regions as defined by the DKT atlas are delineated by colored outlines. The colorbar represents the $-\log _{10}\left(p_{F D R}\right)$ values; so 2.562 (the left hemisphere threshold) is equivalent to $p_{F D R}$ $=10^{-2.562} \approx 0.003$. The $P$ value thresholds are separate for each hemisphere because FDR-adjustment was performed separately. There is extensive overlap with the group differences based on regional mean cortical thickness (c and d). LH, left hemisphere; RH, right hemisphere. 
Table 4. Subcortical and cortical regions $s^{\mathrm{a}}$ of volume $\left(\mathrm{mm}^{3}\right)$ differences between Fontan and control subjects

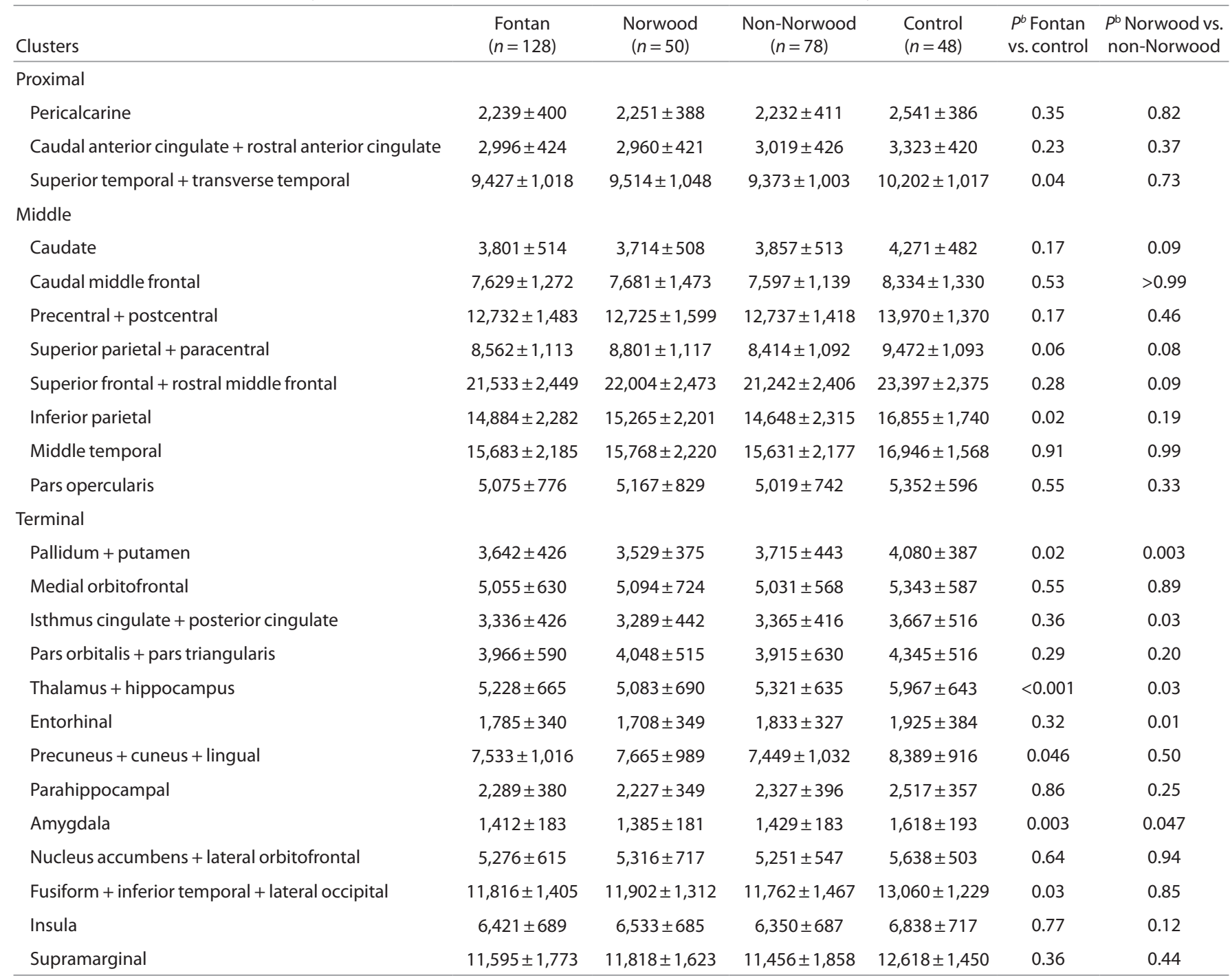

Values are mean \pm SD.

${ }^{a}$ All regions are bilateral.

betermined by linear regression adjusting for age at MRI, sex, field strength, and total intracranial volume.

associated with increased isthmus cingulate-posterior cingulate thickness ( $r=0.37, P=0.004)$. No significant association with characteristics of the reported first operation was noted among Norwood subjects.

Finally, among all Fontan subjects, increasing number of catheterizations was associated with lower isthmus cingulateposterior cingulate cortical thickness $(r=-0.26, P=0.006)$. Increasing number of catheterization complications was associated with lower fusiform-inferior temporal-lateral occipital cluster thickness $(r=-0.25, P=0.009)$.

No association with medical history variables was noted among Norwood subjects. Among non-Norwood subjects, increasing number of catheterization complications was associated with lower parahippocampal thickness $(r=-0.34$, $P=0.005)$.

\section{DISCUSSION}

Consequent to application of quantitative MRI volumetric tools, we report significant and widespread reduction of regional gray matter (GM) volume and cortical thickness in pre-teen and adolescent children born with SV physiology and palliated with the Fontan procedure as compared to healthy control children. Volume reduction was especially prominent in subcortical GM, including pallidum, putamen, thalamus, hippocampus, and amygdala; differences in cortical regions resided predominantly in the temporal and parietal lobes. Remarkably, thinned cortex was widespread, spanning all four major lobes of the brain. In addition, several important surgery-related variables (e.g., older age at first operation, number of catheterizations, and number of operative or catheterization complications) were shown to be associated with reduced GM 


\section{Articles $\mid$ watson e tal.}

Table 5. Cortical regions $s^{\mathrm{a}}$ of thickness $(\mathrm{mm})$ differences between Fontan and control subjects

\begin{tabular}{|c|c|c|c|c|c|c|}
\hline Clusters & $\begin{array}{l}\text { Fontan } \\
(n=115)\end{array}$ & $\begin{array}{l}\text { Norwood } \\
(n=44)\end{array}$ & $\begin{array}{c}\text { Non-Norwood } \\
\quad(n=71)\end{array}$ & $\begin{array}{l}\text { Control } \\
(n=45)\end{array}$ & $\begin{array}{l}P^{b} \text { Fontan vs. } \\
\text { control }\end{array}$ & $\begin{array}{l}P^{\mathrm{b}} \text { Norwood vs. } \\
\text { non-Norwood }\end{array}$ \\
\hline \multicolumn{7}{|l|}{ Proximal } \\
\hline Pericalcarine & $1.68 \pm 0.14$ & $1.70 \pm 0.14$ & $1.67 \pm 0.15$ & $1.72 \pm 0.13$ & 0.51 & 0.46 \\
\hline Caudal anterior cingulate + rostral anterior cingulate & $2.71 \pm 0.14$ & $2.73 \pm 0.13$ & $2.70 \pm 0.15$ & $2.70 \pm 0.13$ & 0.82 & 0.36 \\
\hline Superior temporal + transverse temporal & $2.70 \pm 0.14$ & $2.71 \pm 0.13$ & $2.69 \pm 0.14$ & $2.73 \pm 0.11$ & 0.11 & 0.58 \\
\hline Precentral + postcentral & $2.42 \pm 0.11$ & $2.42 \pm 0.12$ & $2.42 \pm 0.10$ & $2.48 \pm 0.10$ & 0.002 & 0.28 \\
\hline Superior parietal + paracentral & $2.46 \pm 0.14$ & $2.49 \pm 0.14$ & $2.44 \pm 0.14$ & $2.53 \pm 0.12$ & * & 0.21 \\
\hline Superior frontal + rostral middle frontal & $2.68 \pm 0.14$ & $2.70 \pm 0.14$ & $2.67 \pm 0.15$ & $2.72 \pm 0.10$ & 0.03 & 0.62 \\
\hline Inferior parietal & $2.72 \pm 0.17$ & $2.76 \pm 0.16$ & $2.70 \pm 0.17$ & $2.78 \pm 0.15$ & 0.005 & 0.15 \\
\hline Medial orbitofrontal & $2.53 \pm 0.16$ & $2.55 \pm 0.15$ & $2.53 \pm 0.17$ & $2.54 \pm 0.12$ & 0.48 & 0.92 \\
\hline Isthmus cingulate + posterior cingulate & $2.61 \pm 0.16$ & $2.64 \pm 0.16$ & $2.59 \pm 0.15$ & $2.66 \pm 0.14$ & 0.13 & 0.18 \\
\hline Pars orbitalis + pars triangularis & $2.70 \pm 0.14$ & $2.71 \pm 0.13$ & $2.69 \pm 0.15$ & $2.73 \pm 0.12$ & 0.02 & 0.80 \\
\hline Entorhinal & $3.22 \pm 0.29$ & $3.18 \pm 0.31$ & $3.24 \pm 0.27$ & $3.14 \pm 0.29$ & 0.24 & 0.40 \\
\hline Precuneus + cuneus + lingual & $2.21 \pm 0.13$ & $2.23 \pm 0.13$ & $2.19 \pm 0.13$ & $2.28 \pm 0.11$ & * & 0.23 \\
\hline Parahippocampal & $2.69 \pm 0.24$ & $2.68 \pm 0.20$ & $2.69 \pm 0.27$ & $2.81 \pm 0.28$ & 0.009 & 0.66 \\
\hline Lateral orbitofrontal & $2.70 \pm 0.14$ & $2.73 \pm 0.14$ & $2.68 \pm 0.15$ & $2.67 \pm 0.12$ & 0.89 & 0.16 \\
\hline Fusiform + inferior temporal + lateral occipital & $2.68 \pm 0.13$ & $2.71 \pm 0.12$ & $2.67 \pm 0.14$ & $2.73 \pm 0.10$ & 0.01 & 0.42 \\
\hline Insula & $3.12 \pm 0.13$ & $3.14 \pm 0.12$ & $3.10 \pm 0.14$ & $3.11 \pm 0.13$ & 0.98 & 0.28 \\
\hline
\end{tabular}

Values are mean \pm SD.

${ }^{a}$ All regions are bilateral.

Determined by linear regression adjusting for age at MRI, sex, and field strength.

${ }^{*} P<0.001$.

volume and cortical thickness, suggesting that delaying the initial operation and undergoing additional cardiac procedures could pose a long-term risk for brain development in SV patients (13). However, our study design demonstrates association but does not allow for causal inference.

Although this is the first investigation of quantitative GM differences in pre-teenage and adolescent children with $\mathrm{SV}$, evidence of GM deficits in other CHD cohorts has been reported previously. We recently reported subcortical and cortical volume reduction as well as cortical thickness differences in adolescents born with d-transposition of the great arteries (d-TGA) repaired in infancy (20). There was some overlap in the distributions of cortical difference between the d-TGA and SV groups confined to the parietal lobe. While previous study of a heterogeneous group of CHD patients has revealed reduction in total GM volume, cortical surface area, cerebellum, basal ganglia, hippocampus, and thalamus, parcellation of specific cortical gyri and evaluation of cortical thickness was not performed $(25,26)$. Studies of children, adolescents, and young adults with CHD and 22q11.2 deletion syndrome revealed volume reductions in parietal, temporal, and occipital regions, as well as hippocampus $(27,28)$. Our results add to a growing body of work indicating that the structural brain differences seen in utero and in the neonatal period do not necessarily normalize as patients near the end of development and persist in a broad but specifically gyral and subcortical distribution.

Longstanding differences in brain GM seen in adolescence may derive from in utero and perinatal experience (29). Using MRI and cranial ultrasound as early as 18 wk gestation, CHD fetuses-including those with HLHS-were consistently shown to have smaller head circumference (30), increased prevalence of brain abnormalities (9), lower total brain volume (31), and white matter (WM) abnormalities $(9,30)$. Growth rates in head circumference; cerebral total, GM, and WM volumes; and brain metabolic biomarkers are slower for CHD fetuses relative to controls. Importantly, Sun et al. found that brain size at $36 \mathrm{wk}$ gestation correlates positively with both ascending aortic oxygen saturation and cerebral $\mathrm{VO}_{2}$, suggesting that chronic hypoxia in utero is a major contributor to these developmental brain differences (31). Consequently, it is possible that reduced cerebral perfusion or blood oxygen tension, even during the prenatal and preoperative periods, may 
result in cerebral tissue injury that leads to the altered GM volume and thickness that we have found among the SV children in adolescence.

Brain abnormalities present in preoperative studies are often exacerbated after neonatal heart surgery, particularly WM injury (13). Indeed, infants with CHD have brain immaturity similar to that of preterm infants: the WM injury that often manifests in preterm infants, periventricular leukomalacia (PVL), has also been reported in CHD infants and suspected to be caused by hypoxic-ischemic damage $(29,32)$. Although PVL is specific to WM, it can engender consequent neuronal death and disruption of thalamocortical connectivity $(33,34)$. Adolescents born premature have demonstrated lower cortical volumes and thickness bilaterally in several cortical regions $(35,36)$. Notably, GM differences found in d-TGA adolescents, have been found to co-localize with widespread reduction in WM microstructure (37). Patients with SV typically have a more complicated clinical course and require more surgical procedures than d-TGA patients; while correction of d-TGA typically occurs in the neonatal period, SV patients are more likely to have ongoing hemodynamic stresses. Taken together, the GM differences we show in pre-teen children and adolescents with SV could be related to in utero and perinatal/perioperative WM injury with consequent GM disruption through Wallerian degeneration and the effects on developing cortical circuits (29).

Interestingly, Fontan adolescents treated with the Norwood procedure demonstrated several regions in which GM volume was reduced as compared to other Fontan patients. The majority of children who were treated with the Norwood procedure had hypoplastic left heart syndrome (HLHS) while those who were not treated with the Norwood had different types of SV physiology. It is possible that the greater regional reduction among the Norwood patients reflects the hypoxia and relatively reduced cerebral perfusion inherent in HLHS. Alternatively, the children treated with the Norwood had one more major open heart procedure than those who were not and consequently may have been exposed to greater intra- and perioperative risk reflected in the greater reduction in regional brain volume as compared to children who did not receive the Norwood procedure.

The occurrence of stroke in our cohort of pre-teen and adolescent Fontan patients was striking and was found in $12 \%$. Patients with SV and treated with the Fontan procedure possess myriad reasons for increased risk of cerebrovascular events (38). In this regard the cohort reported here differs significantly from d-TGA patients in whom stroke occurs much more rarely. Since SV physiology persists throughout life, clinical or subclinical stroke may have occurred at any time in childhood and contributed to the GM differences observed in the current cohort as compared to controls (39).

The brain volumes and thicknesses that significantly correlated with surgery-related variables were near arterial border zones or in subcortical GM, which are more susceptible to hypoxia- or ischemia-related brain injury; consequently, increasing number of procedures may have an additive effect on resultant GM deficits (40). Adverse events due to cardiac catheterization in the current era are relatively uncommon, but include vascular injury that can lead to stroke and hemorrhage in the brain (41). Finally, while cause of surgical delay is not always apparent and may be due to more severe patient illness, longer time to surgery has been associated with postoperative WM injury in HLHS neonates, and may suggest that the initial palliative procedure should be performed as early as possible (13).

Cortical thickness reflects the arrangement, size, and density of cortical GM components. In particular, it reflects the size of the neuropil composed of axo-dendritic ramifications, a reflection of inputs from and outputs to underlying WM. As a result, cortical thickness constitutes an appealing primary metric for intergroup comparison. Volume represents a derived measurement given by thickness multiplied by surface area. It is possible that the adolescents treated with the Norwood procedure experienced some measure of biological stress that affected deeper, central, subcortical GM as a result of the additional procedure.

This study has limitations. We were unable to scan all subjects on a 3T MRI due to the lack of safety data for some of the cardiac coils. As a result, a higher percentage of Fontan patients were scanned on a 1.5T MRI compared to controls; however, MRI volumetric measurements have good agreement across multiple sites, and we adjusted for this factor in our statistical analyses (42). Second, the date of Fontan procedure spanned many years, the majority from 1995 to 2003, and was not performed at the same center for all patients. While this does not limit our findings in terms of tertiary care center or surgeon, there may be increased associated variability not captured. Although the patient group comprises only those with a diagnosis of SV who underwent the Fontan procedure, there were multiple diagnoses (e.g., HLHS, tricuspid atresia, double outlet right ventricle, heterotaxy, etc.) with differing number of patients in each subgroup. As such, our results may not directly apply to any one SV anatomic group. Finally, this article only reports the results of region-based analysis of GM. Future publications will detail regional WM differences in addition to structural connectivity analyses and their associations with neurodevelopmental measures.

\section{Conclusions}

In conclusion, we show that children and adolescents with SV who underwent the Fontan procedure have widespread brain GM differences compared to control subjects. The differences are extensive and are present in multiple cortical and subcortical regions. Risk factors for reduced GM volume and thickness include older age at first operation, more catheterizations, and more operative or catheterization complications. In light of finding these potentially modifiable risk factors, improvements in patient management may mitigate some deficits in brain development in the Fontan population. 


\section{Articles Watson et al.}

\section{STATEMENT OF FINANCIAL SUPPORT}

This work was supported by funding from the National Heart, Lung, and Blood Institute (R01 HL77681 and HL41786), The Children's Heart Foundation (Chicago), the Farb Family Fund, and the Kostin Family Innovation Fund.

Disclosure: The authors have no conflicts of interest to disclose.

\section{REFERENCES}

1. O'Leary PW 2002 Prevalence, clinical presentation and natural history of patients with single ventricle. Prog Pediatr Cardiol 16:31-38.

2. Anderson JB, Beekman RH 3rd, Kugler JD, et al.; National Pediatric Cardiology Quality Improvement Collaborative. Improvement in interstage survival in a national pediatric cardiology learning network. Circ Cardiovasc Qual Outcomes 2015;8:428-36.

3. Feinstein JA, Benson DW, Dubin AM, et al. Hypoplastic left heart syndrome: current considerations and expectations. J Am Coll Cardiol 2012;59(1 Suppl):S1-42.

4. Hoshino K, Ogawa K, Hishitani T, Kitazawa R, Uehara R. Hypoplastic left heart syndrome: duration of survival without surgical intervention. Am Heart J 1999;137:535-42.

5. Newburger JW, Sleeper LA, Frommelt PC, et al.; Pediatric Heart Network Investigators. Transplantation-free survival and interventions at 3 years in the single ventricle reconstruction trial. Circulation 2014;129:2013-20.

6. Pundi KN, Johnson JN, Dearani JA, et al. 40-year follow-up after the fontan operation: long-term outcomes of 1,052 patients. J Am Coll Cardiol 2015;66:1700-10.

7. Bellinger DC, Watson CG, Rivkin MJ, et al. 2015 Neuropsychological status and structural brain imaging in adolescents with single ventricle who underwent the Fontan procedure. J Am Heart Assoc 4:e002302.

8. Gaynor JW, Ittenbach RF, Gerdes M, et al. Neurodevelopmental outcomes in preschool survivors of the Fontan procedure. J Thorac Cardiovasc Surg 2014;147:1276-82; discussion 1282-1283.e5.

9. Brossard-Racine M, du Plessis AJ, Vezina G, et al. Prevalence and spectrum of in utero structural brain abnormalities in fetuses with complex congenital heart disease. AJNR Am J Neuroradiol 2014;35:1593-9.

10. Kinney HC, Panigrahy A, Newburger JW, Jonas RA, Sleeper LA. Hypoxicischemic brain injury in infants with congenital heart disease dying after cardiac surgery. Acta Neuropathol 2005;110:563-78.

11. von Rhein M, Buchmann A, Hagmann C, et al.; Heart and Brain Research Group. Severe congenital heart defects are associated with global reduction of neonatal brain volumes. J Pediatr 2015;167:1259-63.e1.

12. Claessens NH, Moeskops P, Buchmann A, et al. Delayed cortical gray matter development in neonates with severe congenital heart disease. Pediatr Res 2016;80:668-74.

13. Lynch JM, Buckley EM, Schwab PJ, et al. Time to surgery and preoperative cerebral hemodynamics predict postoperative white matter injury in neonates with hypoplastic left heart syndrome. J Thorac Cardiovasc Surg 2014;148:2181-8.

14. Goldberg CS, Schwartz EM, Brunberg JA, et al. Neurodevelopmental outcome of patients after the fontan operation: a comparison between children with hypoplastic left heart syndrome and other functional single ventricle lesions. J Pediatr 2000;137:646-52.

15. Sarajuuri A, Jokinen E, Mildh L, et al. Neurodevelopmental burden at age 5 years in patients with univentricular heart. Pediatrics 2012;130:e1636-46.

16. Evans AC; Brain Development Cooperative Group. The NIH MRI study of normal brain development. Neuroimage 2006;30:184-202.

17. Fischl B. FreeSurfer. Neuroimage 2012;62:774-81.

18. Klein A, Tourville J. 101 labeled brain images and a consistent human cortical labeling protocol. Front Neurosci 2012;6:171.

19. Tatu L, Moulin T, Bogousslavsky J, Duvernoy H. Arterial territories of the human brain: cerebral hemispheres. Neurology 1998;50:1699-708.

20. Watson CG, Asaro LA, Wypij D, Robertson RL Jr, Newburger JW, Rivkin MJ. Altered gray matter in adolescents with d-transposition of the great arteries. J Pediatr 2016;169:36-43.e1.
21. Ghosh SS, Kakunoori S, Augustinack J, et al. Evaluating the validity of volume-based and surface-based brain image registration for developmental cognitive neuroscience studies in children 4 to 11 years of age. Neuroimage 2010;53:85-93.

22. Hagler DJ Jr, Saygin AP, Sereno MI. Smoothing and cluster thresholding for cortical surface-based group analysis of fMRI data. Neuroimage 2006;33:1093-103.

23. Genovese CR, Lazar NA, Nichols T. Thresholding of statistical maps in functional neuroimaging using the false discovery rate. Neuroimage 2002;15:870-8.

24. Barnes J, Ridgway GR, Bartlett J, et al. Head size, age and gender adjustment in MRI studies: a necessary nuisance? Neuroimage 2010;53:1244-55.

25. von Rhein M, Buchmann A, Hagmann C, et al. Brain volumes predict neurodevelopment in adolescents after surgery for congenital heart disease. Brain 2014;137(Pt 1):268-76.

26. Latal B, Patel P, Liamlahi R, Knirsch W, O'Gorman Tuura R, von Rhein M. Hippocampal volume reduction is associated with intellectual functions in adolescents with congenital heart disease. Pediatr Res 2016;80:531-7.

27. Fountain DM, Schaer M, Mutlu AK, Schneider M, Debbané M, Eliez S. Congenital heart disease is associated with reduced cortical and hippocampal volume in patients with 22q11.2 deletion syndrome. Cortex 2014;57:128-42.

28. Schaer M, Glaser B, Ottet MC, et al. Regional cortical volumes and congenital heart disease: a MRI study in 22q11.2 deletion syndrome. J Neurodev Disord 2010;2:224-34.

29. Volpe JJ. Encephalopathy of congenital heart disease- destructive and developmental effects intertwined. J Pediatr 2014;164:962-5.

30. Hinton RB, Andelfinger G, Sekar P, et al. Prenatal head growth and white matter injury in hypoplastic left heart syndrome. Pediatr Res 2008;64: 364-9.

31. Sun L, Macgowan CK, Sled JG, et al. Reduced fetal cerebral oxygen consumption is associated with smaller brain size in fetuses with congenital heart disease. Circulation 2015;131:1313-23.

32. Galli KK, Zimmerman RA, Jarvik GP, et al. Periventricular leukomalacia is common after neonatal cardiac surgery. J Thorac Cardiovasc Surg 2004; 127:692-704.

33. Inder TE, Huppi PS, Warfield S, et al. Periventricular white matter injury in the premature infant is followed by reduced cerebral cortical gray matter volume at term. Ann Neurol 1999;46:755-60.

34. McQuillen PS, Ferriero DM. Perinatal subplate neuron injury: implications for cortical development and plasticity. Brain Pathol 2005;15: 250-60.

35. Nagy Z, Ashburner J, Andersson J, et al. Structural correlates of preterm birth in the adolescent brain. Pediatrics 2009;124:e964-72.

36. Nagy Z, Lagercrantz H, Hutton C. Effects of preterm birth on cortical thickness measured in adolescence. Cereb Cortex 2011;21:300-6.

37. Rivkin MJ, Watson CG, Scoppettuolo LA, et al. Adolescents with D-transposition of the great arteries repaired in early infancy demonstrate reduced white matter microstructure associated with clinical risk factors. J Thorac Cardiovasc Surg 2013;146:543-9.e1.

38. du Plessis AJ, Chang AC, Wessel DL, et al. Cerebrovascular accidents following the Fontan operation. Pediatr Neurol 1995;12:230-6.

39. Horigome H, Iwasaki N, Anno I, Kurachi S, Kurachi K. Magnetic resonance imaging of the brain and haematological profile in adult cyanotic congenital heart disease without stroke. Heart 2006;92:263-5.

40. Gunn AJ, Bennet L. Fetal hypoxia insults and patterns of brain injury: insights from animal models. Clin Perinatol 2009;36:579-93.

41. Bergersen L, Marshall A, Gauvreau K, et al. Adverse event rates in congenital cardiac catheterization - a multi-center experience. Catheter Cardiovasc Interv 2010;75:389-400.

42. Han X, Jovicich J, Salat D, et al. Reliability of MRI-derived measurements of human cerebral cortical thickness: the effects of field strength, scanner upgrade and manufacturer. Neuroimage 2006;32:180-94. 